\title{
Effects of dezocine on prevention of propofol injection pain: a meta-analysis
}

\author{
Chengmao Zhoul,* \\ Yuting Yang ${ }^{2, *}$ \\ Yu Zhu ${ }^{3}$ \\ Lin Ruan ${ }^{4}$
}

'Department of Anesthesiology, Zhaoqing Medical College, Zhaoqing, ${ }^{2}$ Department of Oncology, The

First People's Hospital of Changde, Changde, ${ }^{3}$ Department of Nursing,

Zhaoqing Medical College, Zhaoqing, ${ }^{4}$ Department of Anesthesiology,

Affiliated Tumor Hospital of Guangxi

Medical University, Nanning, People's

Republic of China

*These authors contributed equally to this work
Correspondence: Lin Ruan

Department of Anesthesiology, Affiliated Tumor Hospital of Guangxi Medical University, No 7I River Road, Nanning 530021, People's Republic of China Fax +86 77I 53I 2000

Email RuanLin187@I63.com
This article was published in the following Dove Press journal: Journal of Pain Research

I June 2017

Number of times this article has been viewed

Objective: The objective of this study was to evaluate the effects of dezocine on the prevention of propofol injection pain.

Materials and methods: We searched for randomized controlled trials (RCTs) of dezocine in preventing propofol injection pain, from inception to April 2016, in PubMed, Embase, Cochrane Library, and CNKI. Next, two reviewers independently screened literature, extracted data, and assessed quality in accordance with the inclusion and exclusion criteria. Finally, RevMan 5.2 software was used to conduct a meta-analysis.

Results: Seven RCTs totaling 630 patients were included in this meta-analysis. The meta-analysis study showed: 1 ) compared with the control group (relative risk [RR] $=0.32,95 \%$ CI $[0.26$, 0.39 ], $P<0.00001$ ), the dezocine group showed a decreasing incidence of propofol injection pain; 2) for severity of propofol injection pain, incidences of mild pain ( $R R=0.55,95 \%$ CI [0.40, 0.75], $P=0.0001)$, moderate pain ( $\mathrm{RR}=0.28,95 \% \mathrm{CI}[0.18,0.43], P<0.00001)$, and severe pain (RR $=0.11,95 \%$ CI $[0.06,0.23], P<0.00001)$ were considerably lower in the dezocine group than in the control group; 3) when comparing the incidence of propofol injection pain in the dezocine group with that of the lidocaine group, no statistically significant differences were found ( $\mathrm{RR}=0.86,95 \% \mathrm{CI}[0.66,1.13], P=0.29)$; and 4) subgroup analysis indicated a significant reduction in the incidence of propofol injection.

Conclusion: Dezocine can both prevent propofol injection pain and mitigate its severity, and its efficacy shows no significant difference from that of lidocaine.

Keywords: dezocine, propofol injection pain, meta-analysis, randomized controlled trials

\section{Introduction}

Propofol injection is widely used in clinical settings both as an anesthetic and as a sedative-hypnotic. However, because of its special ingredients, intravenous propofol injection usually causes local injection pain, $28 \%-90 \%$ incidence in adults and $28 \%-85 \%$ in children. This can lead to hemodynamic variations due to pain and therefore is not conducive to the seamless management of anesthesia. On most occasions, a small dose of lidocaine injected intravenously is able to prevent propofol injection pain. Clinical controversy has ensued however, when it is used with unique or rare local anesthetic. Furthermore, dezocine, as a new narcotic analgesic, is also widely used in clinical anesthesia analgesia, and many clinical trials on dezocine for propofol injection pain have been conducted. In addition, the effect of dezocine on the prevention of propofol injection pain is less disputed than that of lidocaine. Therefore, this meta-analysis was designed to evaluate the effect of dezocine on the prevention of propofol injection pain, so as to provide a more reliable foundation for clinical application. 


\section{Materials and methods} Inclusion and exclusion criteria

Types of research

Randomized controlled trials (RCTs), limited to Chinese and English texts, were used in this study; to qualify, both the dezocine group and the placebo group had to have a minimum of 10 participants.

\section{Study subjects}

General anesthesia patients who had received propofol injections were included in this study.

\section{Interventions}

The study consisted of experimental group (dezocine) and control group (saline or placebo).

\section{Outcome indicators}

The main outcome indicators were incidence of propofol injection pain and severity of propofol injection pain (including mild, moderate, and severe).

\section{Exclusion criteria}

The following were the exclusion criteria: 1) data were only available for the evaluation of propofol injection pain; 2) the outcomes of interest were not reported; 3) studies consisting of a case report only; and 4) studies lacking a controlled group.

\section{Search strategy}

We searched PubMed, Embase, Cochrane Library, and CNKI to find RCTs on the efficacy and safety of dezocine in the prevention of propofol injection pain from inception to April 2016. "Propofol," "injection pain," and "dezocine" were the search terms included.

\section{Literature screening and data extraction}

Two reviewers independently screened the literature and extracted data on the basis of inclusion and exclusion criteria. They then cross-checked one another. The two either discussed or consulted with a third party when there was a disagreement.

\section{Quality evaluation}

We evaluated the methodological quality of included studies according to the risk of bias based on the Cochrane Handbook for Systematic Reviews of Interventions (Version, 5.1.0). ${ }^{1}$ We then used a modified Jadad scale to assess the quality. Evaluation contents included randomization, allocation concealment, blinding (implementers and participants), and incomplete outcome indicators, whether or not details were provided for withdrawals and dropouts.

\section{Statistical analysis}

We conducted the meta-analysis using RevMan 5.2. Enumeration data were expressed as relative risk (RR) and $95 \%$ $\mathrm{CI}$, while measurement data (MD) were represented through weighted mean difference (WMD) and 95\% CI. First, a heterogeneity test was done on included studies via the $\chi^{2}$ test. The test level was $\alpha=0.05$, indicating that heterogeneity existed among the studies when $P \leq 0.05$. Next, quantitative analysis was conducted on heterogeneity with an $I^{2}$ test, and heterogeneity existed when $I^{2} \geq 50 \%$. A fixed-effects model was used to conduct the meta-analysis when there was no heterogeneity. A random-effects model was used when the studies showed more statistical heterogeneity (rather than clinical heterogeneity) or when the differences had no significance. A descriptive analysis approach was used when heterogeneity was too large.

\section{Results}

\section{Literature retrieval results}

A total of 246 articles were retrieved, seven of which, totaling 630 patients, were included in the studies ${ }^{2-8}$ after multiple screenings. Figure 1 shows the literature screening process, and Table 1 indicates the basic characteristics of the included studies.

\section{Methodological quality assessment of included studies}

A randomized group model was used in seven of the included studies, and a blinding method was used in three studies., ${ }^{2,4}$ There were no withdrawals or dropouts, and the data were complete, showing neither selectiveness nor bias. Table 1 shows the Jadad scale.

\section{Meta-analysis outcomes}

Incidence of propofol injection pain

Compared with the control group, all included studies reported incidences of propofol injection pain, covering a total of 630 patients. No studies showed statistical heterogeneity $\left(P=0.18, I^{2}=32 \%\right)$. A fixed-effects model was used to conduct the meta-analysis, and the results indicated that the incidence of propofol injection pain in the dezocine group was lower than that of the control group, and the difference was statistically significant ( $\mathrm{RR}=0.32,95 \% \mathrm{CI}[0.26,0.39]$, $P<0.00001$; Figure 2). 


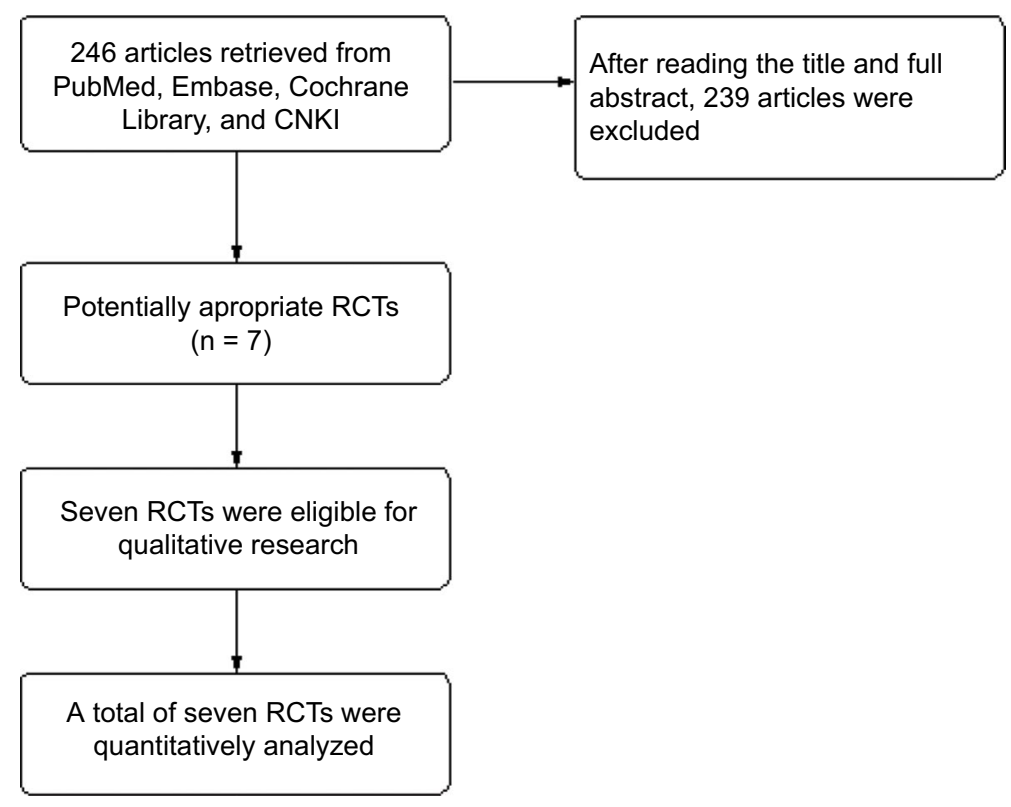

Figure I Flow diagram.

Abbreviation: RCT, randomized controlled trial.

Table I Characteristics of the included studies in the meta-analysis

\begin{tabular}{|c|c|c|c|c|}
\hline Author (published year) & Number of patients & Grouping & Surgical setting & Jadad core \\
\hline Wei and Tongwen $(2014)^{3}$ & 150 & $\begin{array}{l}\text { Normal saline } 3 \mathrm{~mL} \\
2 \% \text { lidocaine } 3 \mathrm{~mL} \\
\text { Dezocine } 0.1 \mathrm{mg} / \mathrm{kg}\end{array}$ & Artificial abortion operation & 4 \\
\hline Jingli and Jinbao $(2013)^{5}$ & 90 & $\begin{array}{l}\text { Normal saline } 2 \mathrm{~mL} \\
2 \% \text { lidocaine } 2 \mathrm{~mL} \\
\text { Dezocine } 2 \mathrm{mg}\end{array}$ & Artificial abortion operation & 4 \\
\hline Ma et al $(2015)^{2}$ & 210 & $\begin{array}{l}\text { Normal saline } 2 \mathrm{~mL} \\
2 \% \text { lidocaine } 2 \mathrm{~mL} \\
\text { Dezocine } 2 \mathrm{mg}\end{array}$ & Elective surgery & 5 \\
\hline Wei and Zaijun (20I2) ${ }^{7}$ & 120 & $\begin{array}{l}\text { Normal saline } 2 \mathrm{~mL} \\
\text { Tramadol } 2 \mathrm{mg} / \mathrm{kg} \\
\text { Dezocine } 2 \mathrm{mg}\end{array}$ & Elective surgery & 4 \\
\hline Qin et al $(2013)^{6}$ & 160 & $\begin{array}{l}\text { Normal saline } 3 \mathrm{~mL} \\
2 \% \text { lidocaine } 30 \mathrm{mg} \\
\text { Fentanyl } 0.1 \mathrm{mg} \\
\text { Dezocine } 10 \mathrm{mg}\end{array}$ & Elective surgery & 4 \\
\hline Xu et al $(2013)^{8}$ & 180 & $\begin{array}{l}\text { Normal saline } 2 \mathrm{~mL} \\
2 \% \text { lidocaine } 2 \mathrm{~mL} \\
\text { Dezocine } 5 \mathrm{mg}\end{array}$ & Elective surgery & 6 \\
\hline Lu et al $(2013)^{4}$ & 75 & $\begin{array}{l}\text { Normal saline } 2 \mathrm{~mL} \\
2 \% \text { lidocaine } 2 \mathrm{~mL} \\
\text { Dezocine } 2 \mathrm{mg}\end{array}$ & Elective surgery & 5 \\
\hline
\end{tabular}

\section{Subgroup analysis}

For analyzing the dose effects of dezocine, we divided the dose into four groups. It appeared that dezocine significantly reduced the incidence of propofol injection pain in all groups, as shown in Figure 3.

Based on the type of surgery in included studies, we divided the studies into two groups. It appeared that dezocine significantly reduced the incidence of propofol injection pain in all groups, as shown in Figure 4.
Compared with the lidocaine group, all included studies, with one exception, reported incidences of propofol injection pain, totaling 550 patients. There was no statistical heterogeneity $\left(P=0.11, I^{2}=45 \%\right)$. We adopted a fixedeffects model to conduct the meta-analysis, showing that the incidence of propofol injection pain in the dezocine group was lower than that of the lidocaine, and no statistical significance was found $(\mathrm{RR}=0.86,95 \% \mathrm{CI}[0.66,1.13]$, $P=0.29$; Figure 5). 


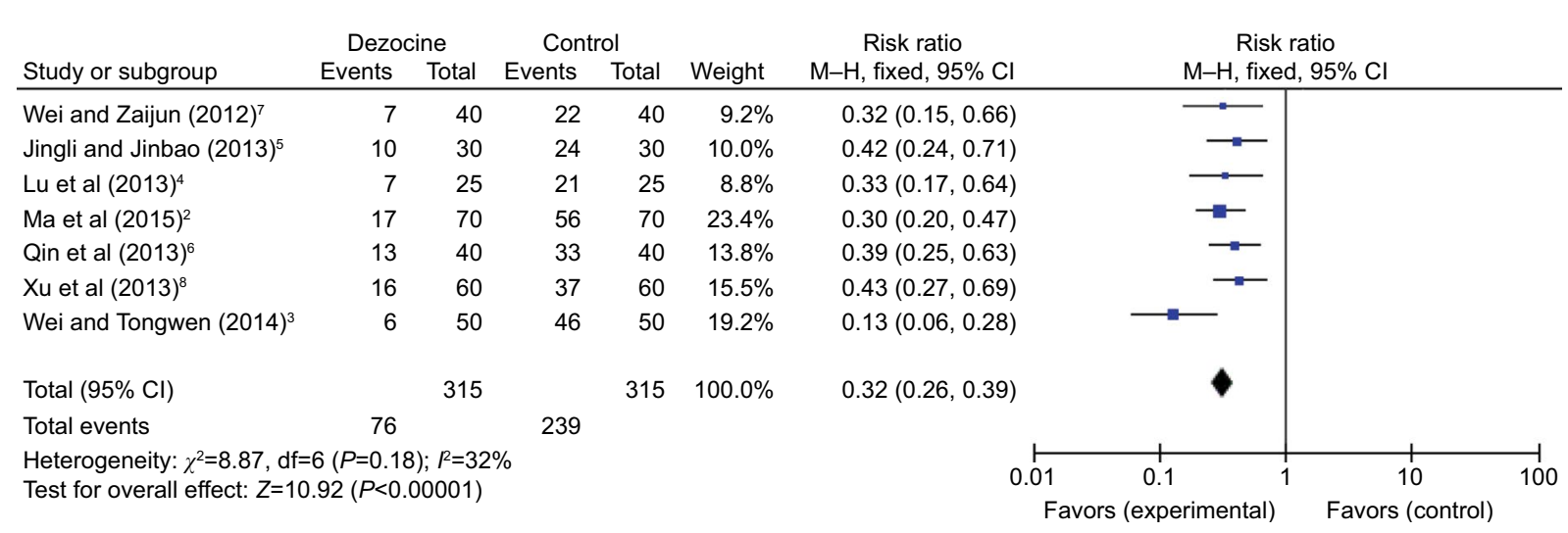

Figure 2 Forest plot of dezocine group and control group on preventing propofol injection pain. Abbreviation: M-H, Mantzel-Haenzel.

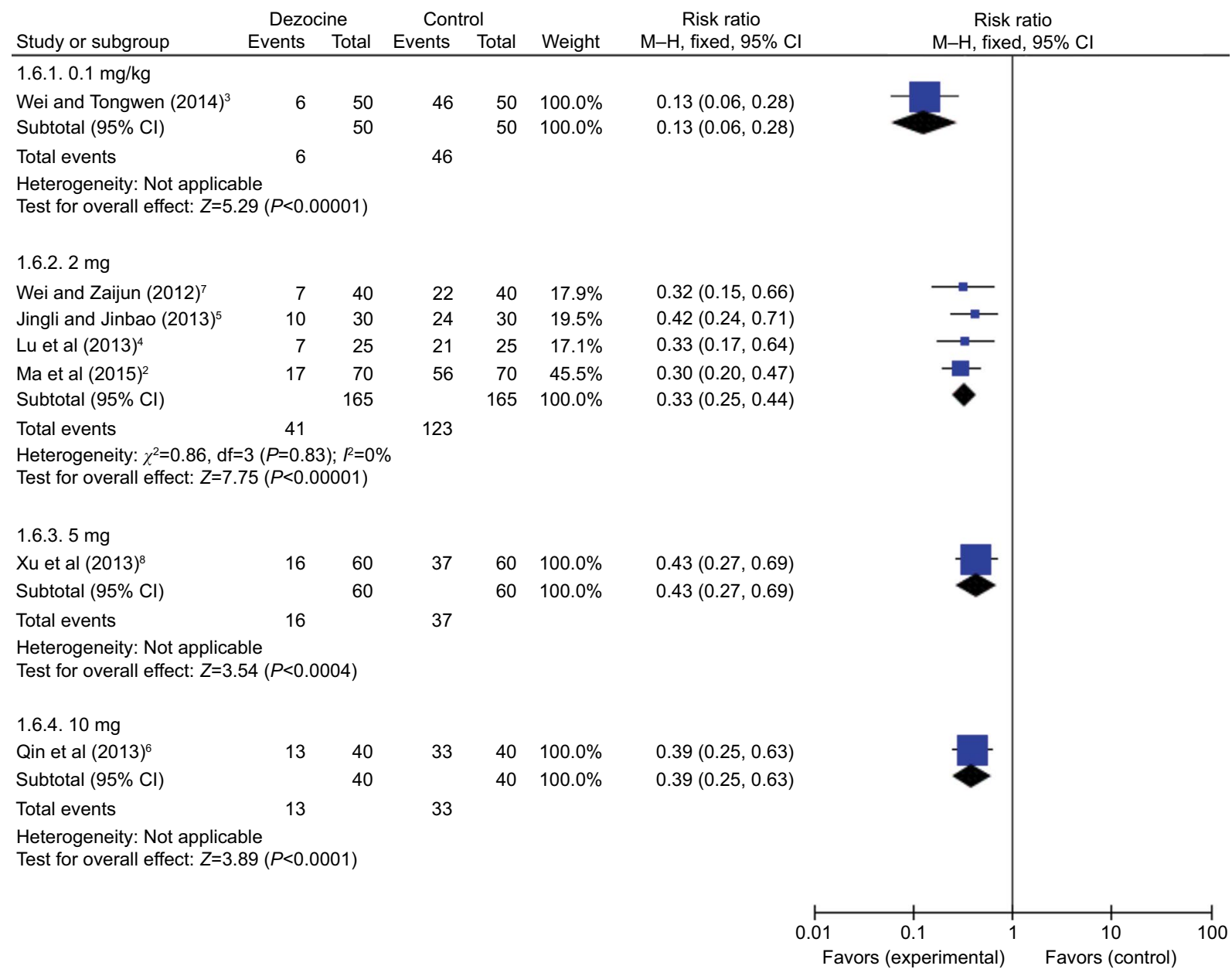

Figure 3 Forest plot of dezocine group and control group on preventing propofol injection pain: a dose subgroup analysis.

Abbreviation: $\mathrm{M}-\mathrm{H}$, Mantzel-Haenzel.

\section{Severity of propofol injection pain} Mild injection pain

All included studies, including a total of 630 patients, reported incidences of mild propofol injection pain, and no statistical heterogeneity $(P=0.80, P=0 \%)$ was found. A fixed-effects model was employed for meta-analysis, and it indicated that incidence of propofol injection pain in the dezocine group was much lower than that in the control group (RR $=0.55,95 \%$ CI [0.40, 0.75], $P=0.0001$; Figure 6).

\section{Moderate pain}

All included studies, including a total of 630 patients, reported incidences of moderate propofol injection pain, and no statistical heterogeneity $\left(P=0.96, P^{2}=0 \%\right)$ was found. 


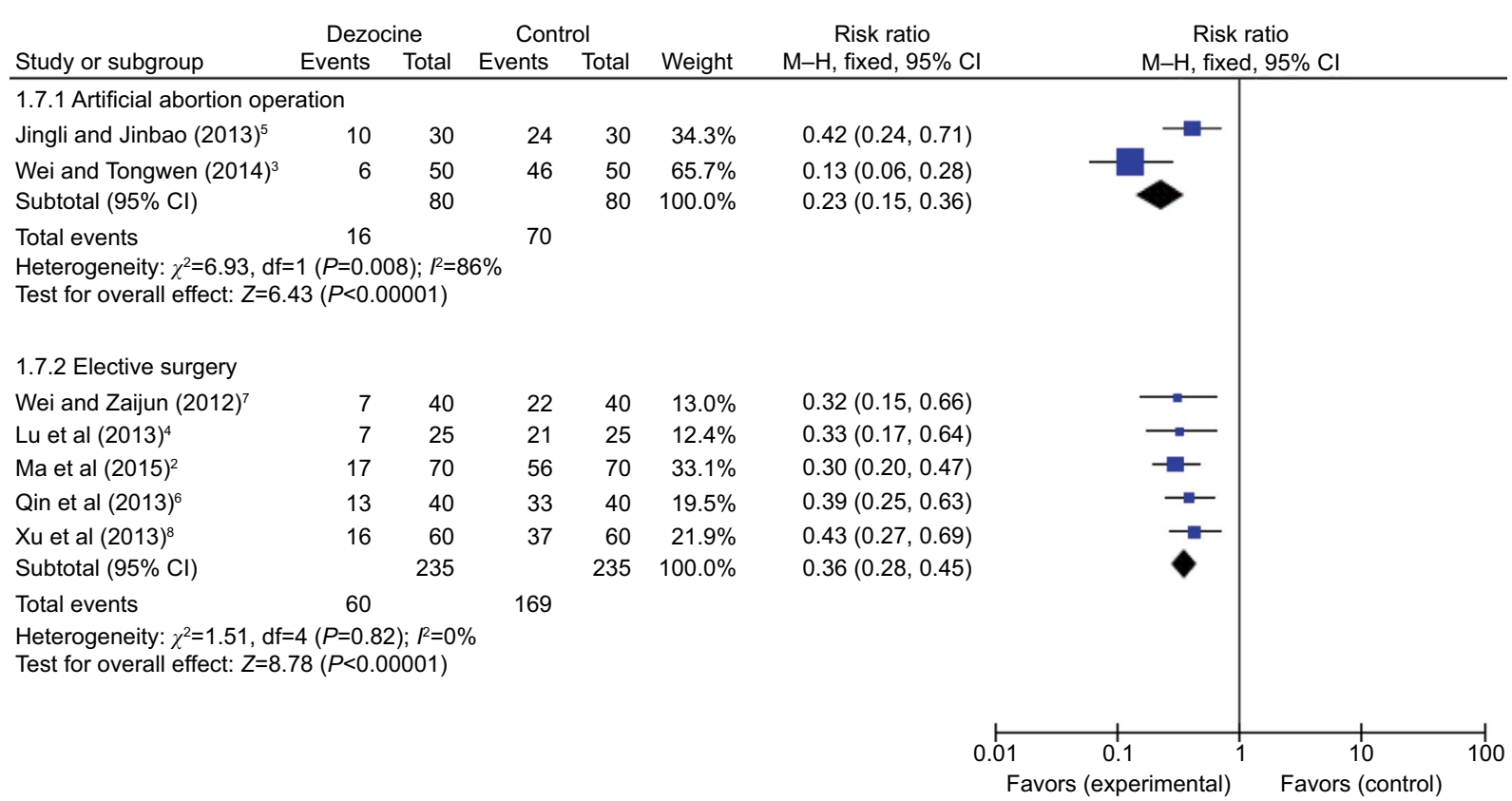

Figure 4 Forest plot of dezocine group and control group on preventing propofol injection pain: a surgery subgroup analysis.

Abbreviation: M-H, Mantzel-Haenzel.

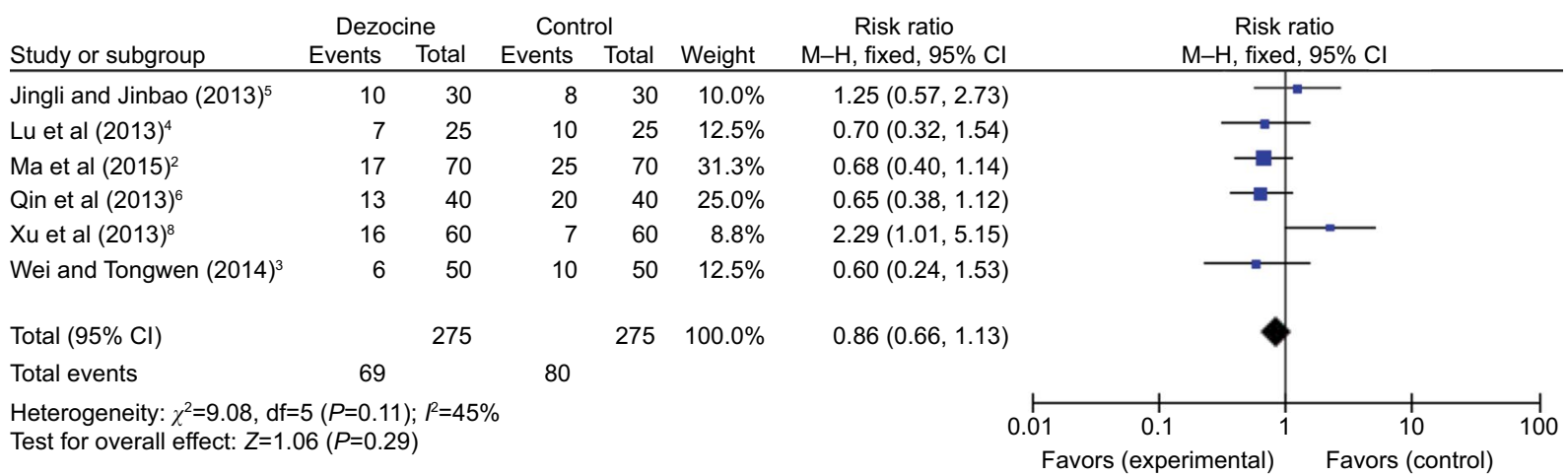

Figure 5 Forest plot of dezocine group and lidocaine group on preventing propofol injection pain. Abbreviation: $\mathrm{M}-\mathrm{H}$, Mantzel-Haenzel.

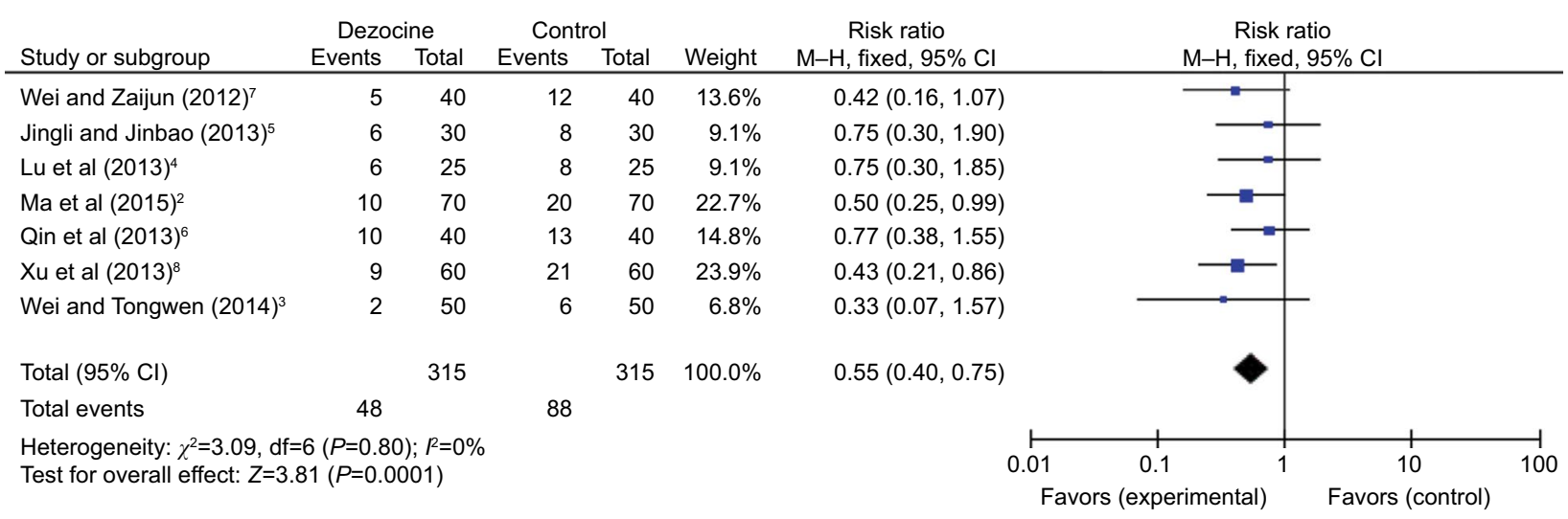

Figure 6 Forest plot of dezocine group and control group on reducing the mild propofol injection pain.

Abbreviation: $\mathrm{M}-\mathrm{H}$, Mantzel-Haenzel.

A fixed-effects model was adopted for meta-analysis, and it showed that the incidence of propofol injection pain in the dezocine group was lower than that in the control group (RR $=0.28,95 \%$ CI $[0.18,0.43], P<0.00001 ;$ Figure 7 ).

\section{Severe pain}

All included studies, including a total of 630 patients, reported incidences of severe propofol injection pain, and no statistical heterogeneity $\left(P=0.14, I^{2}=37 \%\right)$ was found. A 


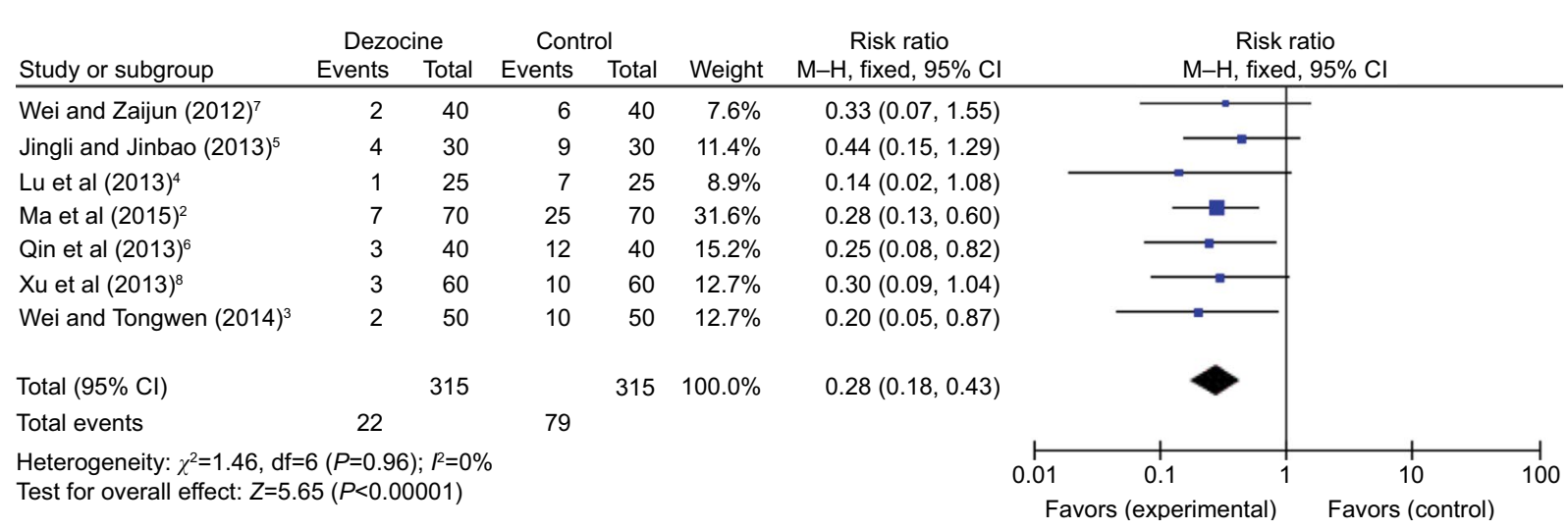

Figure 7 Forest plot of dezocine group and control group on reducing the moderate propofol injection pain. Abbreviation: M-H, Mantzel-Haenzel.

fixed-effects model was used to conduct the meta-analysis, showing that the incidence of propofol injection pain in the dezocine group was lower than that in the control group (RR $=0.11,95 \%$ CI $[0.06,0.23], P<0.00001 ;$ Figure 8$)$.

\section{Sensitivity analysis and funnel plot}

The funnel plot indicated that the results were asymmetrical, showing that there were publication biases. The funnel plot was symmetrical after excluding one RCT. ${ }^{3}$ No statistical heterogeneity ( $P=0.88, I=0 \%$ ) was found. Therefore, we adopted a fixed-effects model to conduct the meta-analysis, and the results showed that the incidence of propofol injection pain in the dezocine group was lower than that in the control group. The results were statistically significant $(\mathrm{RR}=0.36,95 \%$ CI [0.29, 0.45], $P<0.00001$; Figure 9). This result is consistent with those before exclusion, thus showing better stability.

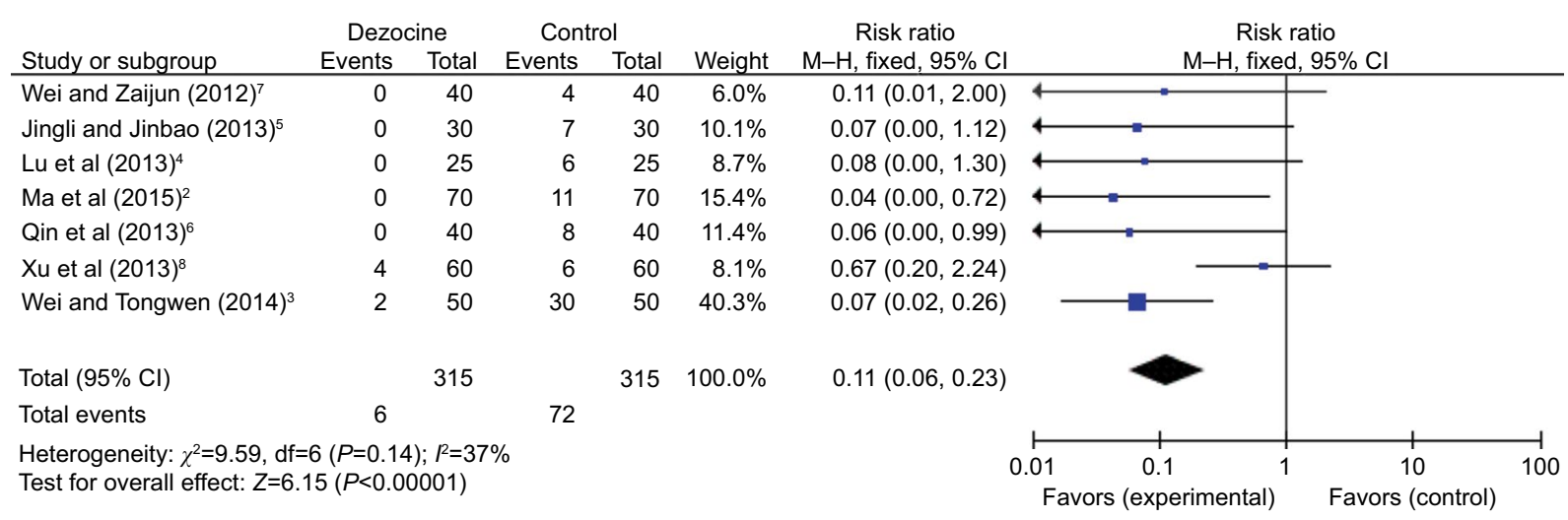

Figure 8 Forest plot of dezocine group and control group on reducing the severe propofol injection pain. Abbreviation: M-H, Mantzel-Haenzel.

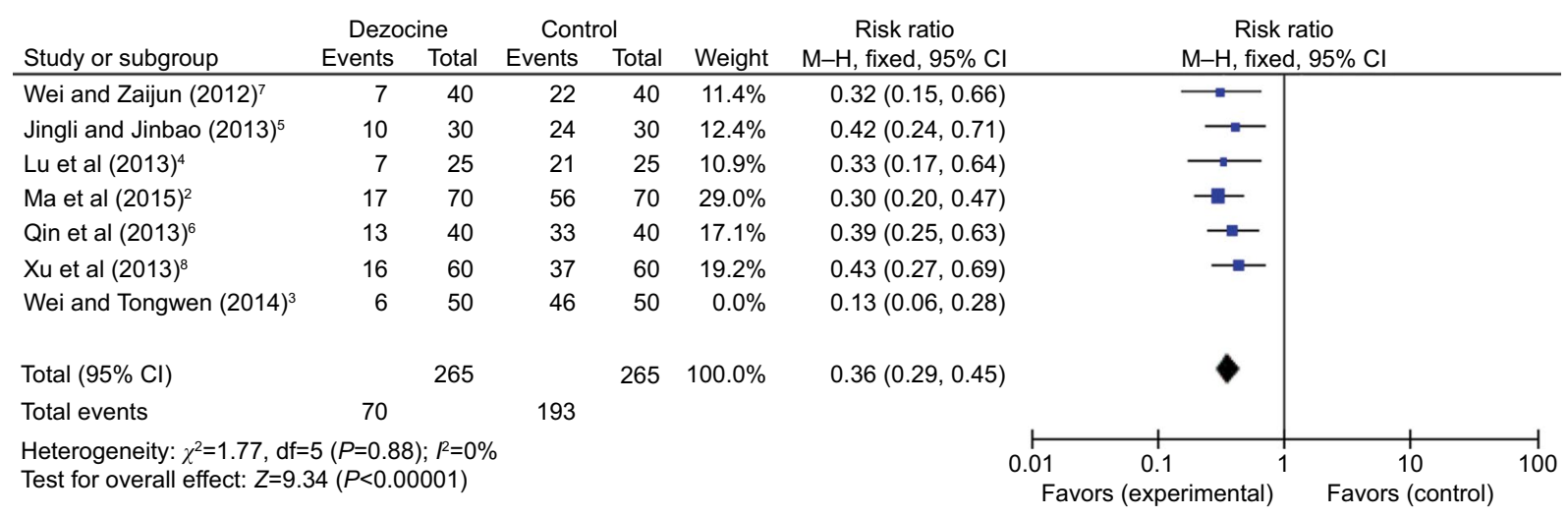

Figure 9 Forest plot of dezocine group and control group. Abbreviation: $\mathrm{M}-\mathrm{H}$, Mantzel-Haenzel. 


\section{Discussion}

This study showed that dezocine can effectively reduce incidences of propofol injection pain, including mild, moderate, and severe pain, and that there was no significance between its effect and that of lidocaine.

Injection pain is a common side effect of propofol in clinical practice. Anesthesiologists have ranked propofol injection pain as No 7 on a list of 33 low-mortality, clinical anesthesia problems, based on its importance and incidence. ${ }^{9}$ Existing research shows that injection pain occurs because the fat solvency of propofol has indirect effects on vascular endothelium. This activates kallikrein in the plasma-kinin system, which then releases histamine, making local venules expand and inducing high permeability. This thereby increases contacts between propofol and free nerve endings. ${ }^{10}$ Presently, the most commonly used method in clinical practice is a combination of drugs to prevent propofol injection pain, of which lidocaine is the most widely used. However, several studies have proven that lidocaine may increase the diameter of propofol particles and thus inflict pulmonary embolism. ${ }^{11}$ Additionally, a combination of lidocaine and propofol may damage the stability of propofol emulsion, thus limiting the effect of lidocaine.

Dezocine, as a $\kappa$ opioid receptor agonist, is also a $\mu$ opioid receptor antagonist. It is a potent opioid analgesic, the analgesic strength, onset time, and duration of which are equal to that of the classical opioid morphine. It can therefore mitigate pain for postoperative patients. This study has found that injecting dezocine in the peripheral vein (PV) can ease propofol injection pain. This may be the result of the combination of dezocine and the central nervous system or opioid receptors in the vascular vessel wall. ${ }^{12}$ This meta-analysis study also showed that there was no difference between the efficacy of dezocine and that of lidocaine in preventing propofol injection pain. Therefore, dezocine can be an ideal alternative to lidocaine in the prevention of propofol injection pain in clinical practice.

There are several limitations to this study. Although the seven included RCTs were included in the dezocine test group, the dezocine dose and the medication time varied.
Among the included studies, only three adopted a blinding method. Thus, process and result measurement bias could not be excluded, and results were also affected. More rigorously designed, detailed, and high-quality RCTs are needed to verify the above conclusions.

\section{Acknowledgment}

The authors are grateful to You-Jing Luo, MD, for her extensive support throughout the article process, which substantially improved the quality of the manuscript.

\section{Disclosure}

The authors report no conflicts of interest in this work.

\section{References}

1. Higgins JPT, Green S. Cochrane Handbook for Systematic Reviews of Interventions. Version 5.1.0; 2011. Available from: www. cochranehandbook.org

2. Ma X, Gao F, Tang L. A Comparison between lidocaine and dezocine on the prevention of propofol injection pain. Heilongjiang Med J. 2015;11:1229-1230.

3. Wei Y, Tongwen Y. The effect of dezocine and lidocaine on pain of propofol-induced injection and effect of artificial abortion analgesia. $J$ North Pharm. 2014;01:12-13.

4. Lu Y, Ye Z, Wong GT, Dong C, Yu J. Prevention of injection pain due to propofol by dezocine: a comparison with lidocaine. Indian J Pharmacol. 2013;45(6):619-621.

5. Jingli L, Jinbao C. The effect of dezocine and lidocaine on pain of propofol-induced injection. Jilin Med J. 2013;19:3840-3841.

6. Qin Z-J, Lan Z-X, Zeng S. Dezocine pretreatment reduces the injection pain of prorofol a prospective, randomized, double-blind, placebo controlled study. Chin J Clin. 2013;41(05):40-42.

7. Wei L, Zaijun H. The effect of dezocine and tramadol on pain of propofol-induced injection. Nei Mongol J Tradit Chin Med. 2012; 15:51-52.

8. Xu X-W, Xing S-L, Li Q-B. Prevention of propofol-induced injection pain: comparison of prophylactic effects between lidocaine and dezocine. Clin J Med Off. 2013;08:816-817.

9. Macario A, Weinger M, Truong P. Which clinical anesthesia outcomes are both common and important to avoid? The perspective of a panel of expert anesthesiologists. Anesth Analg. 1999;88(5):1085-1091.

10. Majedi H, Rabiee M, Khan ZH. A comparison of metoclopramide and lidocaine for preventing pain on injection of diazepam. Anesth Analg. 2002;95(5):1297-1299.

11. Davies AF, Vadodaria B, Hopwood B, Dexter T, Conn D. Efficacy of microfiltration in decreasing propofol-induced pain. Anaesthesia. 2002;57(6):557-561.

12. Li Z, Nie W-J, Li B-J. Expression of opioid ( $\delta, \mu$ and $\kappa)$ receptors in the wall of human blood vessels. Acta Academiae Medicinae Qingdao Universitatis. 2004;3(1):34-36.
Journal of Pain Research

\section{Publish your work in this journal}

The Journal of Pain Research is an international, peer reviewed, open access, online journal that welcomes laboratory and clinical findings in the fields of pain research and the prevention and management of pain. Original research, reviews, symposium reports, hypothesis formation and commentaries are all considered for publication.

\section{Dovepress}

The manuscript management system is completely online and includes a very quick and fair peer-review system, which is all easy to use. Visit http://www.dovepress.com/testimonials.php to read real quotes from published authors. 